\title{
Genotype imputation and variability in polygenic risk score estimation
}

\author{
Shang-Fu Chen ${ }^{1,2}$, Raquel Dias ${ }^{1,2}$, Doug Evans ${ }^{1,2}$, Elias L. Salfati ${ }^{1,2}$, Shuchen Liu ${ }^{1,2}$, Nathan E. Wineinger ${ }^{1,2}$ \\ and Ali Torkamani ${ }^{1,2^{*}}$ (i)
}

\begin{abstract}
Background: Polygenic risk scores (PRSs) are a summarization of an individual's genetic risk for a disease or trait. These scores are being generated in research and commercial settings to study how they may be used to guide healthcare decisions. PRSs should be updated as genetic knowledgebases improve; however, no guidelines exist for their generation or updating.

Methods: Here, we characterize the variability introduced in PRS calculation by a common computational process used in their generation-genotype imputation. We evaluated PRS variability when performing genotype imputation using 3 different pre-phasing tools (Beagle, Eagle, SHAPEIT) and 2 different imputation tools (Beagle, Minimac4), relative to a WGS-based gold standard. Fourteen different PRSs spanning different disease architectures and PRS generation approaches were evaluated.

Results: We find that genotype imputation can introduce variability in calculated PRSs at the individual level without any change to the underlying genetic model. The degree of variability introduced by genotype imputation differs across algorithms, where pre-phasing algorithms with stochastic elements introduce the greatest degree of score variability. In most cases, PRS variability due to imputation is minor $(<5$ percentile rank change) and does not influence the interpretation of the score. PRS percentile fluctuations are also reduced in the more informative tails of the PRS distribution. However, in rare instances, PRS instability at the individual level can result in singular PRS calculations that differ substantially from a whole genome sequence-based gold standard score.

Conclusions: Our study highlights some challenges in applying population genetics tools to individual-level genetic analysis including return of results. Rare individual-level variability events are masked by a high degree of overall score reproducibility at the population level. In order to avoid PRS result fluctuations during updates, we suggest that deterministic imputation processes or the average of multiple iterations of stochastic imputation processes be used to generate and deliver PRS results.
\end{abstract}

Keywords: Genotype phasing, Genotype imputation, Polygenic risk score, PRS, Coronary artery disease, Polygenic score, Genetic risk score, Genome-wide score

\footnotetext{
* Correspondence: atorkama@scripps.edu

${ }^{1}$ Scripps Research Translational Institute, Scripps Research, La Jolla, CA 92037,

USA

2Department of Integrative Structural and Computational Biology, Scripps

Research, La Jolla, CA 92037, USA
}

(c) The Author(s). 2020 Open Access This article is licensed under a Creative Commons Attribution 4.0 International License, which permits use, sharing, adaptation, distribution and reproduction in any medium or format, as long as you give appropriate credit to the original author(s) and the source, provide a link to the Creative Commons licence, and indicate if changes were made. The images or other third party material in this article are included in the article's Creative Commons licence, unless indicated otherwise in a credit line to the material. If material is not included in the article's Creative Commons licence and your intended use is not permitted by statutory regulation or exceeds the permitted use, you will need to obtain permission directly from the copyright holder. To view a copy of this licence, visit http://creativecommons.org/licenses/by/4.0/. The Creative Commons Public Domain Dedication waiver (http://creativecommons.org/publicdomain/zero/1.0/) applies to the data made available in this article, unless otherwise stated in a credit line to the data. 


\section{Background}

Polygenic risk scores (PRSs) are of increasing utility in early risk detection, risk stratification, therapy prioritization, and life-planning [1-3]. In practice, individualized PRS reports should be updated over time as the underlying genetic studies and resultant knowledgebases expand $[4,5]$. This need to refresh PRSs is analogous to the need to update monogenic testing results as underlying knowledgebases of pathogenic variants evolve [6-8]. However, PRSs are continuous scores that typically use the entirety of an underlying knowledgebase for score calculation, whereas monogenic variant analysis and reporting involve the binary (or categorical) reclassification of a constrained set of rare variants present in any individual's genome. As a result, the PRS update process may introduce fluctuations in any and possibly all individual-level scores, whereas updates to monogenic testing results typically involve the rarer reclarification of ambiguous results (the re-classification of a variant of unknown significance). This major difference leads to special considerations for updating of PRSs.

Besides updates to genetic knowledgebases, the most commonly used basic computational approaches for PRS derivation can introduce variability in the resultant score. Currently, sparse genotyping approaches, like array-based SNP genotyping or low-pass whole genome sequencing (WGS), are the dominant assays used to obtain genetic data for PRS calculation. For example, tens of millions of individuals have pre-existing SNP array data acquired from direct-to-consumer genetic testing companies that can and is used to calculate PRSs. These technologies are inexpensive, a feature necessary for population-wide screening where PRSs would be most useful, suggesting sparse genotyping approaches will be the preferred mode of PRS deployment even as WGS costs decline. Sparse genotyping approaches require a genotype imputation step to infer the full set of genotypes included in PRS calculations. This imputation process has been demonstrated to produce scores that are highly correlated overall with PRSs derived from gold standard WGS data [9-12]. However, genotype imputation algorithms can include stochastic elements, which introduce variability in the inferred genotype dosages at the individual level. Here, we investigate the influence of this variability on the stability of individual PRS estimates for multiple genetic diseases with similar results. Results for coronary artery disease $(\mathrm{CAD})$ are presented in the main text as our motivating example because of its emerging utility [13-15]. Results for multiple PRS derivation methods applied to other conditions of interest including atrial fibrillation, breast cancer, type 2 diabetes, glaucoma, and Alzheimer's disease are provided in the Supplementary Files.
Overall, we find that genotype imputation introduces minor fluctuations in PRSs. While small fluctuations in a PRS may not materially influence risk estimates and resultant clinical/personal conclusions, these fluctuations may influence the perceived stability and/or confidence in these estimates. Moreover, the range of these fluctuations varies depending upon the imputation algorithms used, and in rare instances, these fluctuations can result in a score change exceeding 20 percentile points despite no change in the underlying genetic data. These rare large-scale fluctuations are not obvious when PRS reproducibility is evaluated at the population level but can lead to significant differences in the perception and interpretation of PRSs at the individual level. In other rare instances, score fluctuations result in the reclassification of individuals across risk tiers as determined by relative population rank thresholds. These rare score fluctuations would occur in population screening settings where thousands, if not millions, of individuals would receive PRS results and longitudinal updates. Here, we characterize PRS variability at the individual level and suggest the use of imputation algorithms with more deterministic behavior given that overall imputation accuracy is comparable across all algorithms.

\section{Methods}

An overview of the computational process we executed is presented in Fig. 1.

\section{Genetic dataset and pre-processing}

A total of 3574 individuals from the Atherosclerosis Risk in Communities (ARIC) [16] study with both arraybased SNP genotype data and WGS data available were initially retrieved from National Center for Biotechnology Information dbGAP server (phs000280.v6.p1). Samples were genotyped on Genome-Wide Human SNP Array 6.0 chip with genotypes called using Birdseed calling algorithm [17] with samples and SNPs filtered using standard call rate, sex mismatch, and Hardy-Weinberg equilibrium thresholds as described previously [18]. To isolate algorithmic issues from of data format issues, we standardized all genotype data to forward strand GRCh37 orientation as is generated by variant calling from WGS data. Strand orientation was standardized using Strand Home (https://www.well.ox.ac.uk/ wrayner/strand/) strand and build files. Multi-allelic and duplicate variants were excluded using bcftools v1.9 with view (flags: -M 2 -m 2) and norm (flags: -d both) functions [19]. A total of 39,087 variants were excluded in this step. Any strand flips or unresolvable ambiguous variants were detected and corrected or removed using Genotype Harmonizer v1.4.20 with 1000 Genomes Phase3 v5 (1000G) providing the expected linkage disequilibrium (LD) relationships (flags: --callRateFilter 


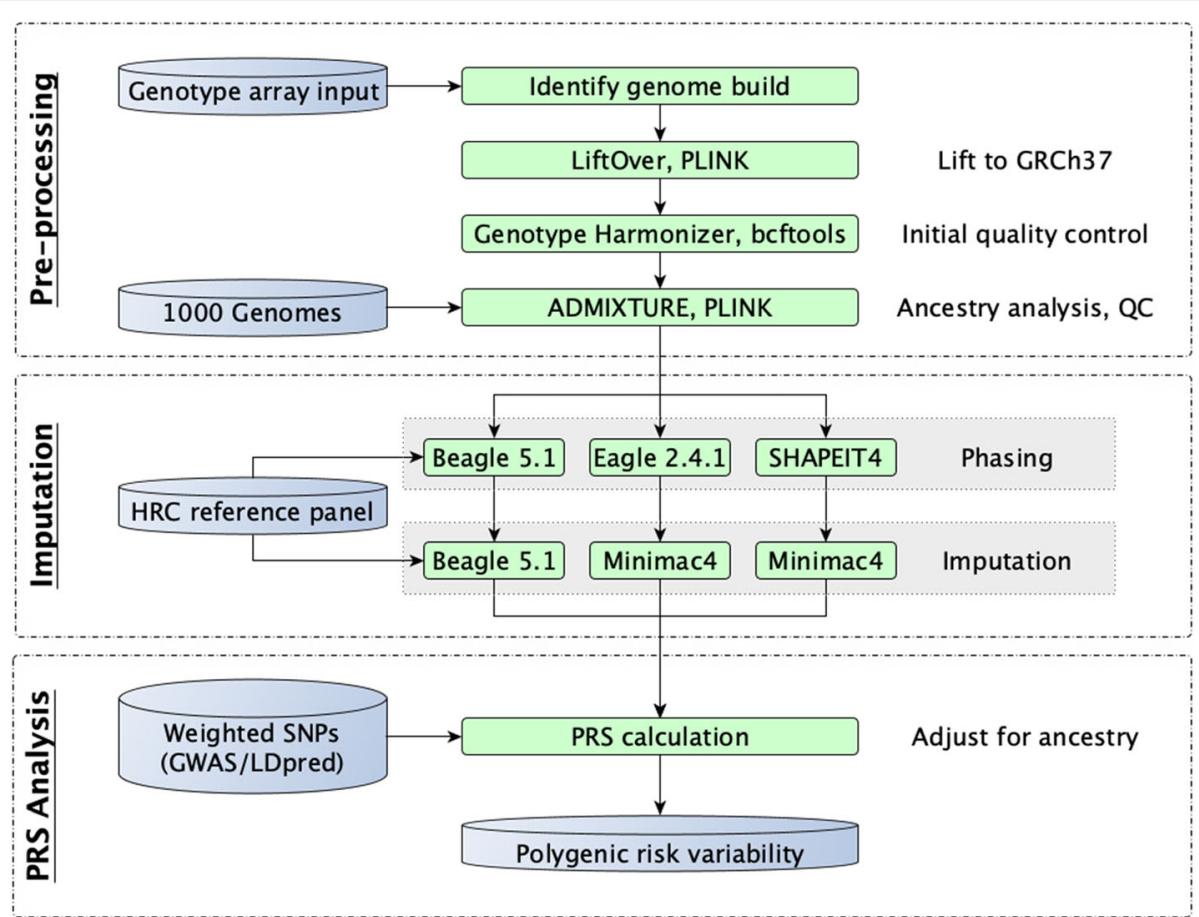

Fig. 1 Study overview. A schematic overview of our polygenic risk computational process. Genetic data was standardized (pre-processing), underwent imputation using three different common genotype imputation processes (imputation), and PRS analysis (PRS analysis)

0.90 -keep). One hundred ninety-seven variants were corrected, and 53,109 variants were removed in this step. The remaining 70,715 strand ambiguous variants were eliminated with bcftools with +fixref plugin (flags: $-\mathrm{m}$ flip -d). A final round of filtering was applied to remaining indels, variants with missing alternative allele information, and multi-allelic variants using bcftools. Sixty variants were removed in this final step. A total of 678,849 variants of 841,820 original variants were retained for imputation.

For gold standard WGS-based variant calls, freeze 3 WGS variant calls were acquired from the Trans-Omics for Precision Medicine program. Variant calls were generated using the GATK best-practices v3.2-2 pipeline from TruSeq PCR-free libraries generated and sequenced at a number of different sequencing sites. For more details of the computational pipelines producing variant calls, see https://www.nhlbiwgs.org/sequencingand-data-processing-methods-freeze3a.

\section{Ancestry estimation}

For ancestry estimation, an independent set of SNPs was defined by LD pruning with PLINK 1.9 (flags: --indeppairwise $100 \quad 10 \quad 0.05$ ); see www.cog-genomics.org/ plink/1.9/ [20]. For each individual, we estimated the $\%$-contribution of each of the five 1000G continental superpopulations to their genetic ancestry using
ADMIXTURE v1.3.0 (flags: --supervised 5) [21]. To isolate the influence of genetic ancestry and admixture on PRS variability, we included only individuals with $>95 \%$ European (EUR) or African (AFR) ancestry in our downstream analysis. This filter resulted in 1447 EUR ancestry individuals and 239 AFR ancestry individuals considered for the ancestry-specific PRS reproducibility analyses.

\section{Phasing and imputation}

We performed phasing and imputation using three commonly used combinations of algorithms: (1) pre-phasing and imputation with Beagle 5.1 [22] (referred to as Beagle), (2) pre-phasing with Eagle v2.4.1 [23] and imputation with Minimac4 [24] (referred to as Eagle+ Minimac), and (3) pre-phasing with SHAPEIT4 [25] and imputation with Minimac4 [24] (referred to as SHAP EIT+Minimac). Minimac and Beagle were run with default settings. For Eagle, we disabled imputation of partially missing genotypes (flags: --noImpMissing) and otherwise applied default settings. With SHAPEIT4, we applied default settings. We used the default genetic map files provided by SHAPEIT4 authors. A total of 37, 995,438 variants were imputed six times per condition to ascertain PRS variability. We performed all phasing and imputation steps using the Haplotype Reference Consortium (HRC) as reference [26]. 


\section{PRS calculation and ancestry adjustment}

PRS calculation was performed using the standard weighted allele-counting approach assuming independent effects across loci using the following equation:

$$
\operatorname{PRS}_{i}=\sum_{j=1}^{n} x_{i j} \beta_{j}
$$

where an individual's $(i)$ PRS is the sum of the effect allele dosages $\left(0 \leq x_{i j} \leq 2\right)$ for each variant $(j)$ included in the score multiplied by its marginal effect size $\left(\beta_{j}-\log \right.$ odds ratio per dosage of effect allele). Percentile ranking was calculated within each matched ancestry members of the study cohort. Specifically, we calculated a 161 SNP score based on a recent analysis identifying 163 confident CAD risk loci [27] with weights based on the latest large-scale CAD meta-GWAS-the CARD IoGRAMplusC4D consortium [28] ( $\left.\mathrm{PRS}_{\mathrm{CAD}}\right)$. The genetic model for non-additive variants was determined as previously described $[29,30]$. SNPs and weights are provided in Additional file 1: Table S1. Two variants were excluded from this list, one multi-allelic variant and one variant absent in HRC, resulting in a final 161 SNP score. One hundred twenty-three of these SNPs are not present in the SNP array data and were imputed. Of the
123 imputed SNPs, 105/123 (85.37\%) were in LD with a genotyped SNP at $R^{2}>0.5,77 / 123(62.60 \%)$ at $R^{2}>0.8$, and $62 / 123(50.41 \%)$ at $R^{2}>0.9$ in EUR populations, and for AFR populations $72 / 123(58.54 \%)$ at $R^{2}>0.5,38 / 123$ $(30.89 \%)$ at $R^{2}>0.8$, and $34 / 123(27.64 \%)$ at $R^{2}>0.9$. In addition, we investigated the variability of two additional previously published PRS scores for CAD, as well as atrial fibrillation, type 2 diabetes, Alzheimer's disease, glaucoma, and breast cancer, derived from a variety score construction methodologies as defined in the PGS Catalog (http://www.pgscatalog.org/browse/studies/). These additional PRS models ranged from dozens to millions of risk loci. The breakdown of the number of SNPs and those directly genotyped vs imputed SNPs is provided in Table 1. All statistical analyses were performed in R v3.5.1.

\section{Imputation accuracy calculation}

For evaluating the imputation accuracy, we developed a flexible python script to calculate the coefficient of determination $\left(R^{2}\right)$ and other accuracy metrics not used here: $F$-score and imputation quality score [38]. The python script for calculating imputation accuracy is available at https://github.com/TorkamaniLab/imputation accuracy_calculator.

Table 1 The breakdown of typed vs imputed SNPs per PRS score

\begin{tabular}{|c|c|c|c|c|c|}
\hline & \multicolumn{4}{|c|}{ Number of SNP } & \multirow[t]{2}{*}{ Reference } \\
\hline & Total & Found & Typed & Imputed & \\
\hline \multicolumn{6}{|c|}{ Coronary artery disease (CAD) } \\
\hline $\mathrm{PRS}_{\mathrm{CAD}}$ & 163 & 161 & 38 & 123 & {$[27]$} \\
\hline metaGRS $_{C A D}$ & $1,745,180$ & $1,736,608$ & 143,724 & $1,592,884$ & {$[31]$} \\
\hline $\mathrm{GPS}_{\mathrm{CAD}}$ & $6,630,150$ & $6,238,460$ & 567,297 & $5,671,163$ & [3] \\
\hline \multicolumn{6}{|l|}{ Type 2 diabetes (T2D) } \\
\hline PRS-GWAS ${ }_{\text {T2D }}$ (547) & 558 & 547 & 66 & 481 & {$[32]$} \\
\hline PRS-GWAST2D $(397)$ & 403 & 397 & 29 & 368 & [33] \\
\hline PRS-GWAS ${ }_{\text {T2D }}(170487)$ & 171,249 & 170,487 & 10,152 & 160,335 & {$[33]$} \\
\hline $\mathrm{GPS}_{\mathrm{T} 2 \mathrm{D}}$ & $6,917,436$ & $6,482,889$ & 570,779 & $5,912,110$ & [3] \\
\hline \multicolumn{6}{|l|}{ Breast cancer (BC) } \\
\hline PRS-GWAS ${ }_{B C}(239)$ & 313 & 239 & 34 & 205 & {$[34]$} \\
\hline PRS-GWAS BC (2935) & 3820 & 2941 & 325 & 2616 & {$[34]$} \\
\hline $\mathrm{GPS}_{\mathrm{BC}}$ & 5218 & 4457 & 374 & 4083 & {$[3]$} \\
\hline \multicolumn{6}{|l|}{ Atrial fibrillation (Afib) } \\
\hline PRS-GWAS ${ }_{\text {Afib }}$ & 166 & 166 & 19 & 147 & {$[35]$} \\
\hline $\mathrm{GPS}_{\text {Afib }}$ & $6,730,541$ & $6,302,924$ & 570,913 & $5,732,011$ & [3] \\
\hline \multicolumn{6}{|l|}{ Alzheimer's disease (AD) } \\
\hline PRS-GWAS & 29 & 29 & 2 & 27 & {$[36]$} \\
\hline \multicolumn{6}{|l|}{ Glaucoma } \\
\hline PRS-GWAS Glaucoma & 2673 & 2657 & 224 & 2433 & {$[37]$} \\
\hline
\end{tabular}




\section{Results}

We evaluated the variability in PRS scores due to 3 common imputation processes (Beagle, Eagle+Minimac, SHAPEIT+Minimac), using 3 different pre-phasing tools (Beagle, Eagle, SHAPEIT) and 2 different imputation tools (Beagle, Minimac4), relative to a WGS-based gold standard (Fig. 1). Here, we present the variability observed across the combined cohort of 1686 individuals of $>95 \%$ European ancestry (1447 individuals) or $>95 \%$ African ancestry (229 individuals). Plots separated by genetic ancestry are provided in Supplemental Figuresthose results are consistent with the combined results presented here, with a greater degree of variability observed in the African ancestry sub-group.

For each imputation process, we repeated the PRS calculation process six times starting from the pre-phasing step. Imputation-based PRSs were generally highly correlated with ground truth WGS-based PRSs (average $R^{2}>0.9$ ), except for the Alzheimer's disease PRS and two type 2 diabetes PRSs which consistently showed lower correlation with WGS-based ground truth (Fig. 2a, Additional file 1: Table S2, Fig. S1A-S13A). For each score, the overall level of score dispersion relative to the
WGS-based gold standard is approximately equivalent across all three imputation processes when evaluated at the population level-being primarily associated with the genetic architecture being imputed. However, when we evaluate the range of PRS percentile values achieved across the different imputation methods at an individual level, clear differences in PRS stability emerge; SHAP EIT+Minimac leads to the most intra-individual variability, followed by Beagle and Eagle+Minimac (Fig. 2b, Additional file 2: Fig. S1B-S13B). In rare instances $(\sim 1 \%$ of the time), SHAPEIT+Minimac results in a range of $>$ 20 percentile points between the highest and lowest PRS values achieved for an individual (Table 2, Additional file 1: Table S3). Beagle and Eagle+Minimac return relatively stable PRS values, with both methods very rarely $(\sim$ $0.1 \%)$ resulting in PRS values that change $>10$ percentile points between the highest and lowest values achieved for an individual. This algorithm-level variability is observed regardless of the original approach used to derive the PRS and the number of SNPs included in the score-as similar variability is observed for three different CAD risk scores derived using very different strategies and including vastly different numbers of SNPs

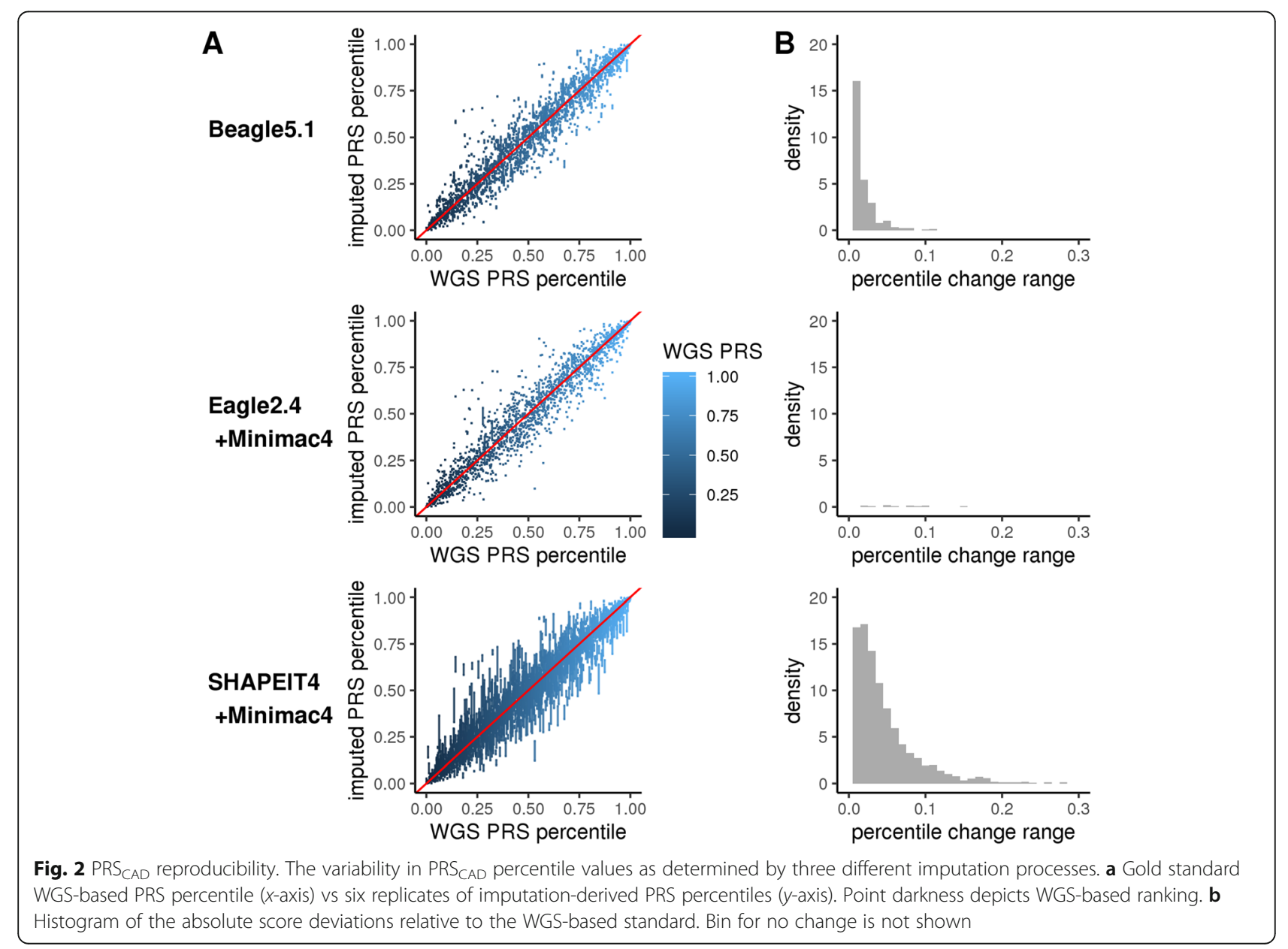


Table 2 The distribution of CAD risk score percentile changes caused by three different imputation processes

\begin{tabular}{|c|c|c|c|c|c|c|c|c|c|}
\hline \multirow{2}{*}{$\begin{array}{l}\text { Percentile } \\
\text { change }\end{array}$} & \multicolumn{3}{|l|}{ Beagle } & \multicolumn{3}{|c|}{ Eagle+Minimac } & \multicolumn{3}{|c|}{ SHAPEIT+Minimac } \\
\hline & EUR & AFR & All & EUR & AFR & All & EUR & AFR & All \\
\hline \multicolumn{10}{|l|}{ PRS $_{\text {CAD }}(161)$} \\
\hline$\leq 1 \%$ tile & 1247 (86.18\%) & $163(68.2 \%)$ & 1410 (83.63\%) & 1437 (99.31\%) & 236 (98.74\%) & 1673 (99.23\%) & $259(17.9 \%)$ & $5(2.09 \%)$ & $264(15.66 \%)$ \\
\hline$>1 \%$ tile & $200(13.82 \%)$ & 76 (31.8\%) & 276 (16.37\%) & $10(0.69 \%)$ & $3(1.26 \%)$ & $13(0.77 \%)$ & 1188 (82.1\%) & 234 (97.91\%) & $1422(84.34 \%)$ \\
\hline > 5\%tile & $21(1.45 \%)$ & $5(2.09 \%)$ & $26(1.54 \%)$ & $7(0.48 \%)$ & $1(0.42 \%)$ & $8(0.47 \%)$ & 331 (22.87\%) & 168 (70.29\%) & 499 (29.6\%) \\
\hline$>10 \%$ tile & $2(0.14 \%)$ & $0(0 \%)$ & $2(0.12 \%)$ & $1(0.07 \%)$ & $0(0 \%)$ & $1(0.06 \%)$ & $68(4.7 \%)$ & 87 (36.4\%) & 155 (9.19\%) \\
\hline$>20 \%$ tile & $0(0 \%)$ & $0(0 \%)$ & $0(0 \%)$ & $0(0 \%)$ & $0(0 \%)$ & $0(0 \%)$ & $3(0.21 \%)$ & $10(4.18 \%)$ & $13(0.77 \%)$ \\
\hline \multicolumn{10}{|c|}{ metaGRS $_{\text {CAD }}(1736608)$} \\
\hline$\leq 1 \%$ tile & 940 (64.96\%) & $106(44.35 \%)$ & 1046 (62.04\%) & $1418(98 \%)$ & 229 (95.82\%) & 1647 (97.69\%) & 153 (10.57\%) & $13(5.44 \%)$ & 166 (9.85\%) \\
\hline$>1 \%$ tile & 507 (35.04\%) & $133(55.65 \%)$ & $640(37.96 \%)$ & $29(2 \%)$ & $10(4.18 \%)$ & 39 (2.31\%) & $1294(89.43 \%)$ & $226(94.56 \%)$ & $1520(90.15 \%)$ \\
\hline > 5\%tile & $12(0.83 \%)$ & $2(0.84 \%)$ & $14(0.83 \%)$ & $5(0.35 \%)$ & $1(0.42 \%)$ & $6(0.36 \%)$ & 277 (19.14\%) & 154 (64.44\%) & $431(25.56 \%)$ \\
\hline$>10 \%$ tile & $2(0.14 \%)$ & $0(0 \%)$ & $2(0.12 \%)$ & $0(0 \%)$ & $0(0 \%)$ & $0(0 \%)$ & $8(0.55 \%)$ & $42(17.57 \%)$ & $50(2.97 \%)$ \\
\hline$>20 \%$ tile & $0(0 \%)$ & $0(0 \%)$ & $0(0 \%)$ & $0(0 \%)$ & $0(0 \%)$ & $0(0 \%)$ & $0(0 \%)$ & $0(0 \%)$ & $0(0 \%)$ \\
\hline \multicolumn{10}{|c|}{ GPS $_{\text {CAD }}(6238460)$} \\
\hline$\leq 1 \%$ tile & 1289 (89.08\%) & $191(79.92 \%)$ & $1480(87.78 \%)$ & 1429 (98.76\%) & 233 (97.49\%) & 1662 (98.58\%) & $328(22.67 \%)$ & $20(8.37 \%)$ & 348 (20.64\%) \\
\hline$>1 \%$ tile & 158 (10.92\%) & $48(20.08 \%)$ & $206(12.22 \%)$ & $18(1.24 \%)$ & $6(2.51 \%)$ & $24(1.42 \%)$ & 1119 (77.33\%) & 219 (91.63\%) & $1338(79.36 \%)$ \\
\hline > 5\%tile & $26(1.8 \%)$ & $0(0 \%)$ & $26(1.54 \%)$ & $2(0.14 \%)$ & $0(0 \%)$ & $2(0.12 \%)$ & 239 (16.52\%) & $69(28.87 \%)$ & 308 (18.27\%) \\
\hline$>10 \%$ tile & $12(0.83 \%)$ & $0(0 \%)$ & $12(0.71 \%)$ & $0(0 \%)$ & $0(0 \%)$ & $0(0 \%)$ & 56 (3.87\%) & $9(3.77 \%)$ & 65 (3.86\%) \\
\hline$>20 \%$ tile & $2(0.14 \%)$ & $0(0 \%)$ & $2(0.12 \%)$ & $0(0 \%)$ & $0(0 \%)$ & $0(0 \%)$ & $6(0.41 \%)$ & $1(0.42 \%)$ & $7(0.42 \%)$ \\
\hline
\end{tabular}

Columns indicate the number and percentage of subjects in the population with percentile change in their PRS. EUR European, AFR African

(Table 2), as well as across all diseases considered (Fig. 3). These results suggest that the pre-phasing step introduces the bulk of the stochasticity in imputation and PRS results. Variability is further increased in individuals of African ancestry (Table 2, Additional file 2: Fig. S14S27) and is exacerbated for PRSs with worse overall concordance with the WGS-based gold standard.

Next, we determined which individuals are most likely to be affected by imputation-induced PRS score variability. As expected, the variability of the PRS percentile is greatest in the middle of the distribution and lowest at the tails of the distribution when quantified as the average absolute deviation (Fig. 4a, Additional file 1: Fig. S28A-S40A) and maximum absolute deviation (Fig. 4b, Additional file 1: Fig. S28B-S40B) in PRS percentile per person. This is true for both European and African ancestry individuals (Additional file 1: Fig. S41-S54). This is the expected behavior given raw score increments due to imputation variability would be uniform across the normal distribution of the raw PRS, but those equalsized raw score increments result in larger percentile rank changes in the middle of the distribution vs the tails. Thus, the largest percentile rank changes occur in individuals where the utility implications are least influenced by that change (individuals who are overall at average risk and whose score fluctuates within the average risk range). While relatively large percentile changes may not change the utility conclusion for average risk individuals, those changes would likely lead to a perceived change in risk. In addition, we observe some significant variability, $>5$ percentile changes in score, at the tails of the risk distribution. Smaller percentile changes at the extreme ends of the risk distribution can influence both the clinical implications as well as perceptions of risk [3]. Overall, using typical quintile thresholds for defining high (top 20\%) vs intermediate (middle 60\%) vs low (bottom 20\%) risk individuals, we observe that runto-run imputation variability can lead to differing utility conclusions in $<1 \%$ of individuals when using Beagle and Eagle+Minimac, whereas interpretation variability can exceed $5 \%$ of individuals for SHAPEIT+Minimac (Table 3, Additional file 1: Table S4). When evaluated using more refined risk tier thresholds for high-risk individuals, the rate of re-classification increases further as the distance between risk tier thresholds shrinks, in some cases exceeding re-classification of $20 \%$ of individuals placed in a more narrowly defined top/bottom $5 \%$ percentile threshold (Table 4, Additional file 1: Table S5).

Finally, we sought to determine whether imputation score variability was reflective of any differences in overall score accuracy at the genotype level relative to gold standard WGS-based genotypes. We compared the mean and minimum $R^{2}$ values between imputed and gold standard genotype dosages per imputation process and replicate for all SNPs on chromosome 22 (Fig. 5a, b). Overall, the three approaches demonstrate equivalent 


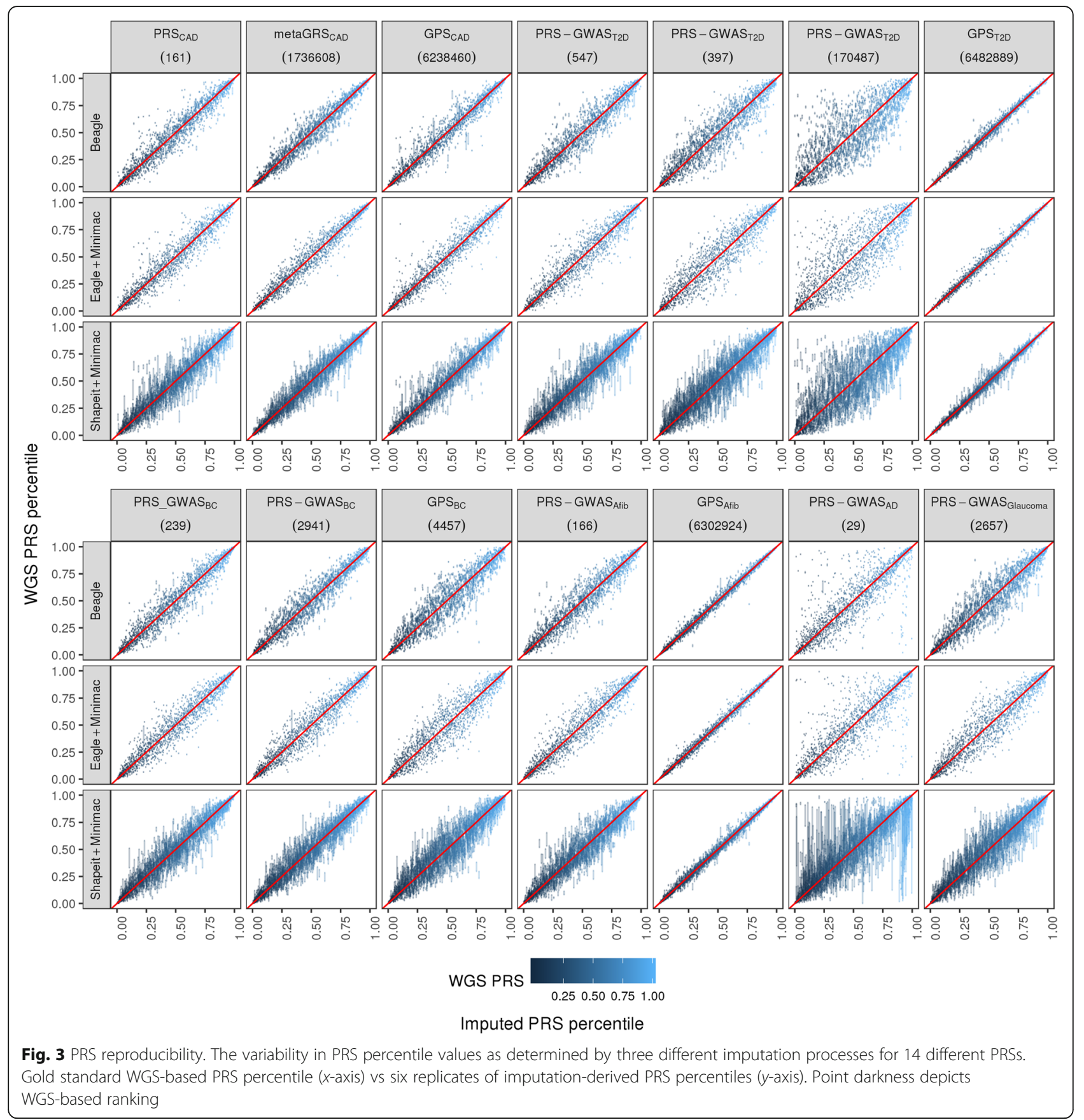

average accuracy relative to WGS-based genotypes $R^{2}=$ $\sim 0.925$ (Fig. 5a left). However, when restricting our view to the minimum accuracy achieved per individual by each process, a left shift in minimum accuracy is observed for SHAPEIT+Minimac (Fig. 5a right) $(p$ value $<$ $2 \mathrm{e}-16$ by paired Wilcoxon). As expected, the range of accuracy achieved is larger for rare genetic variants though variants across the entire minor allele frequency spectrum contribute to this variability in accuracy (Fig. 5b). This individual-level imputation variability is masked by the approximately equivalent average accuracy when evaluated at a population level (Fig. 5a). To dissect the source of variability further, we examined the potential contribution of each SNP to the overall degree of score variability, which is related to the consistency of its imputation (captured by the Gini coefficient of imputation results, which ranges from 0 to 1 where a value of 0 corresponds to completely consistent imputation results) and its relative contribution to the overall score (captured by percent variability explained, which is further a function of the SNP weight and its allele frequency variance) (Fig. 5c). As expected, the vast 


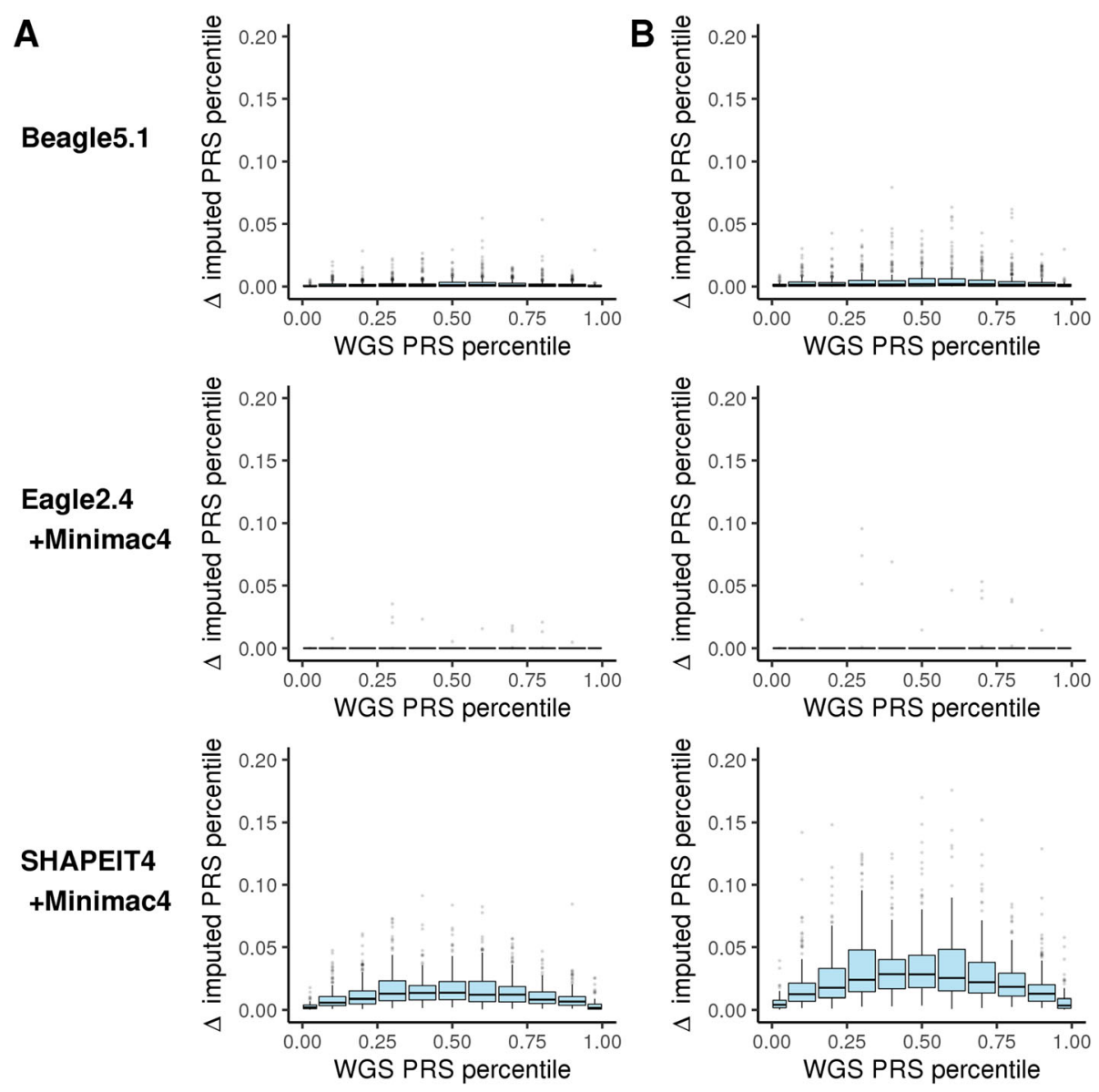

Fig. 4 PRS $C$ AD variability as a function of PRS bin. The degree of variability in PRS percentile as a function of the expected WGS-based PRS tier across three different imputation processes. a Average absolute deviation per individual relative to their WGS-based gold standard. b Maximum absolute deviation per individual relative to the WGS-based gold standard. Box plots depict the interquartile range as is standard

Table 3 The rate of risk tier re-classification due to imputation variability using quintile-based cutoffs

\begin{tabular}{|c|c|c|c|c|c|c|c|c|c|}
\hline \multirow[t]{2}{*}{ Risk tier } & \multicolumn{3}{|l|}{ Beagle } & \multicolumn{3}{|c|}{ Eagle+Minimac } & \multicolumn{3}{|c|}{ SHAPEIT+Minimac } \\
\hline & $<20 \%$ tile & $20-80 \%$ tile & $>80 \%$ tile & $<20 \%$ tile & $20-80 \%$ tile & $>80 \%$ tile & $<20 \%$ tile & 20-80\%tile & $>80 \%$ tile \\
\hline \multicolumn{10}{|l|}{$\mathrm{PRS}_{\mathrm{CAD}}(161)$} \\
\hline$<20 \%$ tile & $19.89 \%$ & $0.09 \%$ & $0 \%$ & $19.99 \%$ & $0 \%$ & $0 \%$ & $19.27 \%$ & $0.72 \%$ & $0 \%$ \\
\hline 20-80\%tile & $0.04 \%$ & $59.90 \%$ & $0.09 \%$ & $0 \%$ & $60.01 \%$ & $0.01 \%$ & $0.48 \%$ & $58.88 \%$ & $0.64 \%$ \\
\hline$>80 \%$ tile & $0 \%$ & $0.10 \%$ & $19.90 \%$ & $0 \%$ & $0.01 \%$ & $19.98 \%$ & $0 \%$ & $0.48 \%$ & $19.52 \%$ \\
\hline \multicolumn{10}{|c|}{ metaGRS $_{\text {CAD }}(1736608)$} \\
\hline$<20 \%$ tile & $19.88 \%$ & $0.11 \%$ & $0 \%$ & $19.98 \%$ & $0.01 \%$ & $0 \%$ & $19.47 \%$ & $0.51 \%$ & $0 \%$ \\
\hline 20-80\%tile & $0.29 \%$ & $59.59 \%$ & $0.13 \%$ & $0.01 \%$ & $60 \%$ & $0 \%$ & $0.51 \%$ & $58.95 \%$ & $0.54 \%$ \\
\hline$>80 \%$ tile & $0 \%$ & $0.09 \%$ & $19.92 \%$ & $0 \%$ & $0.01 \%$ & $19.99 \%$ & $0 \%$ & $0.50 \%$ & $19.50 \%$ \\
\hline \multicolumn{10}{|c|}{$\mathrm{GPS}_{\mathrm{CAD}}(6238460)$} \\
\hline$<20 \%$ tile & $19.85 \%$ & $0.14 \%$ & $0 \%$ & $19.96 \%$ & $0.02 \%$ & $0 \%$ & $19.57 \%$ & $0.42 \%$ & $0 \%$ \\
\hline 20-80\%tile & $0.08 \%$ & $59.76 \%$ & $0.17 \%$ & $0.03 \%$ & $60 \%$ & $0 \%$ & $0.59 \%$ & $58.93 \%$ & $0.48 \%$ \\
\hline$>80 \%$ tile & $0 \%$ & $0.13 \%$ & $19.88 \%$ & $0 \%$ & $0 \%$ & $19.99 \%$ & $0 \%$ & $0.56 \%$ & $19.44 \%$ \\
\hline
\end{tabular}

The rate of re-classification into low risk ( $<20$ th percentile), intermediate risk (20-80th percentile), and high risk ( $>80$ th percentile) due to phasing and imputation variability across all individuals included in this study (1447 EURs and 239 AFRs combined). Columns indicate the average individual-level imputation-based risk tier. Rows indicate the process-level imputation-based risk tier 
Table 4 The rate of risk tier re-classification for high risk individuals due to phasing and imputation variability

\begin{tabular}{|c|c|c|c|}
\hline Risk tier & Beagle & Eagle+Minimac & SHAPEIT+Minimac \\
\hline \multicolumn{4}{|l|}{ PRS $_{\text {CAD }}(161)$} \\
\hline$<5 \%$ tile & $1.19 \%(-1.13-3.51)$ & $0 \%(0-0)$ & $15.66 \%(7.84-23.48)$ \\
\hline$<10 \%$ tile & $2.96 \%(0.4-5.51)$ & $0 \%(0-0)$ & $10.12 \%(5.56-14.68)$ \\
\hline$<15 \%$ tile & $1.59 \%(0.04-3.13)$ & $0 \%(0-0)$ & $9.96 \%(6.26-13.67)$ \\
\hline$<20 \%$ tile & $0.89 \%(-0.11-1.9)$ & $0 \%(0-0)$ & $9.91 \%(6.7-13.12)$ \\
\hline$>80 \%$ tile & $1.78 \%(0.37-3.19)$ & $0.3 \%(-0.28-0.88)$ & $10 \%(6.81-13.19)$ \\
\hline$>85 \%$ tile & $1.98 \%(0.26-3.69)$ & $0 \%(0-0)$ & $12.7 \%(8.59-16.81)$ \\
\hline > 90\%tile & $1.19 \%(-0.45-2.83)$ & $0 \%(0-0)$ & $17.54 \%(11.84-23.24$ \\
\hline > 95\%tile & $4.71 \%(0.2-9.21)$ & $0 \%(0-0)$ & $15.85 \%$ (7.95-23.76) \\
\hline \multicolumn{4}{|c|}{ metaGRS $_{\text {CAD }}(1736608)$} \\
\hline$<5 \%$ tile & $6.98 \%(1.59-12.36)$ & $1.19 \%(-1.13-3.51)$ & 18.6\% (10.38-26.83) \\
\hline$<10 \%$ tile & $2.37 \%(0.07-4.66)$ & $0 \%(0-0)$ & $6.67 \%(2.86-10.47)$ \\
\hline$<15 \%$ tile & $2.37 \%(0.5-4.25)$ & $0.79 \%(-0.3-1.88)$ & $12.94 \%(8.82-17.06)$ \\
\hline$<20 \%$ tile & $3.82 \%(1.79-5.86)$ & $0.3 \%(-0.28-0.88)$ & $8.9 \%(5.86-11.94)$ \\
\hline$>80 \%$ tile & $2.66 \%(0.95-4.38)$ & $0 \%(0-0)$ & $9.76 \%(6.6-12.93)$ \\
\hline$>85 \%$ tile & $4.72 \%(2.12-7.33)$ & $0 \%(0-0)$ & $12.11 \%(8.11-16.11)$ \\
\hline > 90\%tile & $6.43 \%(2.76-10.11)$ & $0 \%(0-0)$ & $6.06 \%(2.42-9.7)$ \\
\hline > 95\%tile & $2.38 \%(-0.88-5.64)$ & $0 \%(0-0)$ & $9.52 \%(3.25-15.8)$ \\
\hline \multicolumn{4}{|c|}{$\operatorname{GPS}_{\mathrm{CAD}}(6238460)$} \\
\hline$<5 \%$ tile & $5.88 \%(0.88-10.88)$ & $0 \%(0-0)$ & $13.25 \%(5.96-20.55)$ \\
\hline$<10 \%$ tile & $2.38 \%(0.08-4.69)$ & $0.59 \%(-0.56-1.75)$ & $7.78 \%$ (3.72-11.85) \\
\hline$<15 \%$ tile & $0.79 \%(-0.3-1.88)$ & $0 \%(0-0)$ & $9.92 \%(6.23-13.61)$ \\
\hline$<20 \%$ tile & $1.49 \%(0.19-2.78)$ & $0.3 \%(-0.28-0.88)$ & $10 \%(6.81-13.19)$ \\
\hline$>80 \%$ tile & $2.37 \%(0.75-3.99)$ & $0 \%(0-0)$ & $8.33 \%(5.38-11.29)$ \\
\hline$>85 \%$ tile & $1.97 \%(0.26-3.68)$ & $0 \%(0-0)$ & $6.48 \%(3.41-9.55)$ \\
\hline > 90\%tile & $2.37 \%(0.07-4.66)$ & $0 \%(0-0)$ & $5.99 \%(2.39-9.59)$ \\
\hline > 95\%tile & $1.18 \%(-1.12-3.47)$ & $0 \%(0-0)$ & $10.59 \%(4.05-17.13)$ \\
\hline
\end{tabular}

The rate of individuals re-classified from high risk tiers due to imputation variability across all individuals included in this study (1447 EURs and 239 AFRs combined). An individual is considered re-classified if at least two imputation replicates produce a different risk tier than the WGS-based ground truth. Values in parenthesis present the $95 \%$ confidence interval

majority of SNPs show low variability as captured by a low Gini coefficient. For those SNPs contributing to score variability, we observe both single SNP variability events (top left plot quadrant-high weight variants with moderate levels of imputation variability like LPA and ANRIL for CAD and APOE for Alzheimer's disease) as well as the accumulation of errors across multiple SNPs (bottom right plot quadrantseveral low weight variants with a higher degree of imputation variability). When applied across a large population, where rare computational events will accumulate in a small subset of individuals simply by random chance, these fluctuations can lead to dramatic PRS differences when the score is updated, even when no changes are made to the underlying PRS model or input genotype data.

\section{Discussion}

Several initiatives are ongoing to evaluate the behavioral and health outcomes associated with PRS reporting [3943]. PRSs will continue to be updated over time as GWASs expand in size, additional risk loci are identified, and more sophisticated score calculation and variant weighting schemes are introduced. While updating our MyGeneRank CAD score (https://mygenerank.scripps. edu/blog/post/mgr-new-update), we sought to identify a computational process that would lead to the greatest degree of PRS stability and accuracy in the face of a score that is expected to evolve over time. In most cases, the degree of score fluctuation resulting from imputation variability is small and does not change the interpretation of the result. Most individuals remain in the same broad risk tier, and the degree of score fluctuation is 


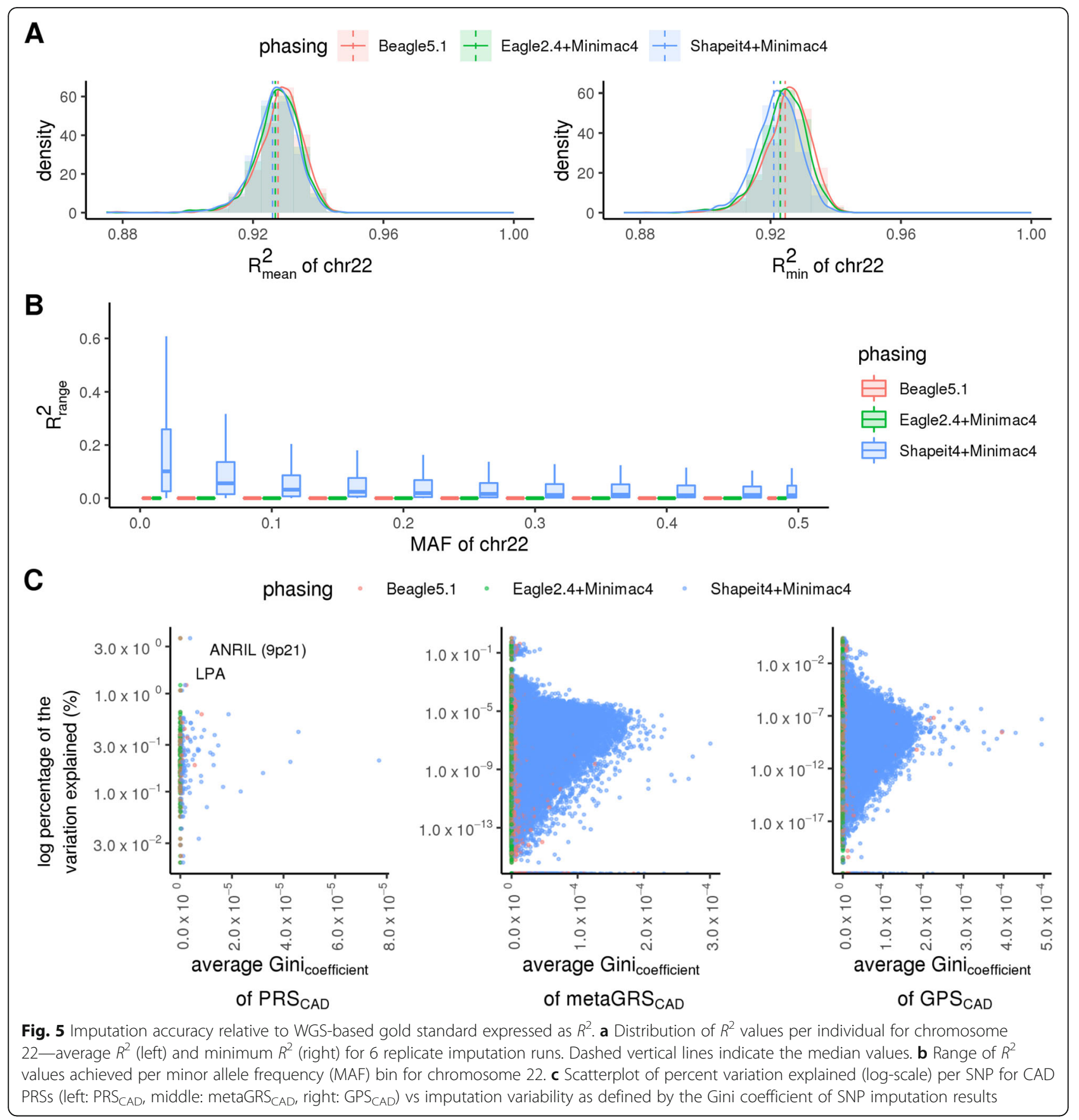

reduced in the tails of the distribution where disease risk implications are most useful. However, upon systematic investigation of the individual-level run-to-run variability of PRS score generation reported here, we observe rare instances where a score update can lead to a substantial fluctuation in PRS without any change to the underlying genetic model. The magnitude of these fluctuations is not obviously related to the method used to define the score nor the number of SNPs contained within a score. Our observations suggest that imputation variability across all components of a score contributes to score fluctuations at an individual level. Though clearly, SNPs with the highest weights contribute most strongly and consistently to score fluctuations under modest but generally accepted levels of imputation variability. In a population screening setting, both infrequent SNP variability events for singular high weight SNPs or the rare accumulation of run-to-run imputation fluctuations across low weight SNPs will return rare score results for some individuals. Given that some of the promise of PRSs in practice relates to prioritization of preventative behaviors, even minor fluctuations in score may reduce 
the perceived confidence of the score by the recipientdespite the fact that the overall risk implications are unchanged. Thus, we suggest that either deterministic imputation processes should be favored or stochastic imputation processes could be run multiple times in order to select the most common result. We expect score accuracy is equivalent under these two scenarios.

\section{Conclusions}

Overall, given the dynamic and evolving nature of PRSs and their purported influence on health decisionmaking, we suggest that methods for the effective forecasting of score changes should be developed. Besides the score variability introduced by computational processes, PRSs also evolve over time depending upon the underlying genetic architecture and size of GWASs currently executed. A projection of the remaining risk loci and their likely effect sizes can be used to project the likely future state of an individual's PRS [44] to provide them some idea of the uncertainty in their risk estimate and to set expectations for the level of score change that might be expected upon update. To accomplish this, all technical sources of score variability must be addressed and minimized in order to allow for an accurate projection of PRS uncertainty due to undiscovered risk loci. During our CAD score update, we found that both computational stochasticity and changes to the underlying genetic model contribute to PRS variability. Score variability due to imputation was expected to be a more prominent issue for PRSs with a small number of contributing variants (like our original 57 SNP MyGeneRank CAD score) and was expected to diminish in impact for PRSs composed of many SNPs, i.e., imputation variability should regress to the mean with high SNP counts. However, our results suggest that significant (and equivalent) score variability exists even in scores composed of thousands of SNPs, suggesting that imputation variability in the most highly weighted SNPs must be carefully addressed in all PRS deployments. Increased imputation variability in underrepresented populations will further exacerbate this issue and potentially contribute to health disparities as a result of PRS deployment [45]. This variability highlights a pressing need for expanded and diverse imputation reference panels. We expect score variability is further exacerbated for admixed individuals especially when considering the additional variability that might be introduced upon matching an admixed individual to a genetically matched reference population to derive relative score rankings. Thus, while updating genetic models themselves introduces the bulk of population-level score variability, it is important to control for computational variability so that score changes and projections due to improvements to PRS knowledgebases can be deployed and communicated with confidence.

\section{Supplementary Information}

The online version contains supplementary material available at https://doi. org/10.1186/s13073-020-00801-x.

Additional file 1: Supplementary Tables S1-S5. Components of PRS CAD (Table S1). Correlation of imputed-based PRSs and WGS-based ground truth (Table S2). The distribution of risk score percentile rank changes caused by three different imputation processes for other diseases (Table S3). The rate of re-classification due to imputation variability at different quintile-based risk tiers (Table S4). The rate of re-classification due to imputation variability for high-risk tiers (Table S5).

Additional file 2: Supplemental Fig. S1-S13. PRS Reproducibility Across Diseases and Score Derivation Methods. Supplemental Fig. S14S27. PRS Reproducibility Across Diseases and Score Derivation Methods by Ancestry. Supplemental Fig. S28-S40. PRS Variability Across Diseases and Score Derivation Methods as a Function of Percentile Bin. Supplemental Fig. S41-S54. PRS Variability Across Diseases and Score Derivation Methods as a Function of Percentile Bin by Ancestry. Supplemental Fig. S55. SNP level variability by Score Impact Across Diseases and Score Derivation Methods.

\section{Abbreviations}

1000G: 1000 Genomes Phase3 v5; AFR: African; ARIC: Atherosclerosis Risk in Communities; CAD: Coronary artery disease; EUR: European; GWAS: Genomewide association study; HRC: Haplotype Reference Consortium; LD: Linkage disequilibrium; PRS: Polygenic risk score; SNP: Single-nucleotide polymorphism; WGS: Whole genome sequencing

\section{Acknowledgements}

We would like to thank J.C. Ducom, Lisa Dong, and the Scripps High Performance Computing service for their support.

\section{Authors' contributions}

SC analyzed and interpreted the study data, created the software, and contributed to the manuscript writing. RD designed the study, created the software, analyzed and interpreted the study data, and contributed to the manuscript writing. DE analyzed and interpreted the study data and created the software. ELS analyzed and interpreted the study data. SL created the software. NE contributed to the study design. AT conceived and designed the study, analyzed and interpreted the study data, and contributed to the manuscript writing. All authors read and approved the final manuscript.

\section{Funding}

This work is supported by R01HG010881 to AT as well as grants U24TR002306 and UL1TR002550.

\section{Availability of data and materials}

The data that support the findings of this study are available from dbGAP, but restrictions apply to the availability of these data, which were used under ethics approval for the current study, and so are not openly available to the public. Data are however available from the authors upon reasonable request and with permission of dbGAP. The ARIC cohort dataset used in this study is available via dbGAP under accession phs000280.v6.p1; https://www. ncbi.nlm.nih.gov/projects/gap/cgi-bin/study.cgi?study_id=phs000280.v6.p1 [46]. The python script for calculating imputation accuracy is available at https:// github.com/TorkamaniLab/imputation_accuracy_calculator [47].

\section{Ethics approval and consent to participate}

Data was downloaded from dbGAP under a Scripps Institutional Review Board protocol IRB-17-7005. Informed consent was obtained by the original ARIC study investigators. This research conforms with the principles of the Helsinki Declaration

\section{Consent for publication}

Individuals were consented for publication under the original ARIC study protocols.

\section{Competing interests}

AT declares he is co-founder of geneXwell. The remaining authors declare that they have no competing interests. 
Received: 23 March 2020 Accepted: 9 November 2020

Published online: 23 November 2020

\section{References}

1. Torkamani A, Wineinger NE, Topol EJ. The personal and clinical utility of polygenic risk scores. Nat Rev Genet. 2018;19:581-90.

2. Lambert SA, Abraham G, Inouye M. Towards clinical utility of polygenic risk scores. Hum Mol Genet. 2019;28(R2):R133-42.

3. Khera AV, Chaffin M, Aragam KG, Haas ME, Roselli C, Choi SH, et al. Genome-wide polygenic scores for common diseases identify individuals with risk equivalent to monogenic mutations. Nat Genet. 2018:50(9):1219-24.

4. Visscher PM, Wray NR, Zhang Q, Sklar P, McCarthy Ml, Brown MA, et al. 10 years of GWAS discovery: biology, function, and translation. Am J Human Genetics. 2017;101:5-22.

5. Chatterjee N, Shi J, García-Closas M. Developing and evaluating polygenic risk prediction models for stratified disease prevention. Nat Rev Genet. 2016; 17(7):392-406

6. Salfati EL, Spencer EG, Topol SE, Muse ED, Rueda M, Lucas JR, et al. Reanalysis of whole-exome sequencing data uncovers novel diagnostic variants and improves molecular diagnostic yields for sudden death and idiopathic diseases. Genome Med. 2019;11:83.

7. Liu P, Meng L, Normand EA, Xia F, Song X, Ghazi A, et al. Reanalysis of Clinical Exome Sequencing Data. N Engl J Med. 2019;380:2478-80.

8. Eldomery MK, Coban-Akdemir Z, Harel T, Rosenfeld JA, Gambin T, StrayPedersen $A$, et al. Lessons learned from additional research analyses of unsolved clinical exome cases. Genome Med. 2017;9:1-15.

9. Wasik K, Berisa T, Pickrell JK, Li JH, Fraser DJ, King K, et al. Comparing lowpass sequencing and genotyping for trait mapping in pharmacogenetics. bioRxiv. 2019;632141. Available from: http://biorxiv.org/content/early/2019/ 05/08/632141.abstract.

10. Pasaniuc B, Rohland N, McLaren PJ, Garimella K, Zaitlen N, Li H, et al. Extremely low-coverage sequencing and imputation increases power for genome-wide association studies. Nat Genet. 2012;44:631-5.

11. Homburger JR, Neben CL, Mishne G, Zhou AY, Kathiresan S, Khera A V. Low coverage whole genome sequencing enables accurate assessment of common variants and calculation of genome-wide polygenic scores. Genome Med. 2019;11:716977.

12. Gilly A, Southam L, Suveges D, Kuchenbaecker K, Moore R, Melloni GEM, et al. Very low-depth whole-genome sequencing in complex trait association studies. Bioinformatics. 2019;35:2555-61.

13. Tikkanen E, Havulinna AS, Palotie A, Salomaa V, Ripatti S. Genetic risk prediction and a 2-stage risk screening strategy for coronary heart disease. Arterioscler Thromb Vasc Biol. 2013;33(9):2261-6.

14. Mega JL, Stitziel NO, Smith JG, Chasman DI, Caulfield MJ, Devlin JJ, et al. Genetic risk, coronary heart disease events, and the clinical benefit of statin therapy: An analysis of primary and secondary prevention trials. Lancet. 2015;385:2264-71.

15. Natarajan P, Young R, Stitziel NO, Padmanabhan S, Baber U, Mehran R, et al. Polygenic risk score identifies subgroup with higher burden of atherosclerosis and greater relative benefit from statin therapy in the primary prevention setting. Circulation. 2017;135(22):2091-101.

16. Investigators TA. The Atherosis Risk in Communities (ARIC) study: design and objectives. Am J Epidemiol. 1989.

17. Korn JM, Kuruvilla FG, McCarroll SA, Wysoker A, Nemesh J, Cawley S, et al. Integrated genotype calling and association analysis of SNPS, common copy number polymorphisms and rare CNVs. Nat Genet. 2008;40(10):1253-60.

18. Psaty BM, O'Donnell CJ, Gudnason V, Lunetta KL, Folsom AR, Rotter II, et al. Cohorts for Heart and Aging Research in Genomic Epidemiology (CHARGE) Consortium design of prospective meta-analyses of genome-wide association studies from 5 Cohorts. Circulation: Cardiovascular Genetics. 2009:2:73-80

19. Li H. A statistical framework for SNP calling, mutation discovery, association mapping and population genetical parameter estimation from sequencing data. Bioinformatics. 2011:27(21):2987-93.

20. Chang CC, Chow CC, Tellier LC, Vattikuti S, Purcell SM, Lee JJ. Secondgeneration PLINK: rising to the challenge of larger and richer datasets. Gigascience. 2015;4(1):7.

21. Alexander DH, Novembre J, Lange K. Fast model-based estimation of ancestry in unrelated individuals. Genome Res. 2009;19:1655-64.
22. Browning $B L$, Zhou $Y$, Browning SR. A one-penny imputed genome from next-generation reference panels. Am J Hum Genet. 2018;103(3):338-48.

23. Loh PR, Danecek P, Palamara PF, Fuchsberger C, Reshef YA, Finucane HK, et al. Reference-based phasing using the Haplotype Reference Consortium panel. Nat Genet. 2016:48:1443-8.

24. Das S, Forer L, Schönherr S, Sidore C, Locke AE, Kwong A, et al. Nextgeneration genotype imputation service and methods. Nat Genet. 2016; 48(10):1284-7.

25. Delaneau O, Marchini J, McVeanh GA, Donnelly P, Lunter G, Marchini JL, et al. Integrating sequence and array data to create an improved 1000 Genomes Project haplotype reference panel. Nat Commun. 2014;5(1):1-9.

26. McCarthy S, Das S, Kretzschmar W, Delaneau O, Wood AR, Teumer A, et al. A reference panel of 64,976 haplotypes for genotype imputation. Nat Genet. 2016;48(10):1279-83.

27. Erdmann J, Kessler T, Munoz Venegas L, Schunkert H. A decade of genomewide association studies for coronary artery disease: The challenges ahead. Cardiovasc Res. 2018:114:1241-57.

28. Nelson CP, Goel A, Butterworth AS, Kanoni S, Webb TR, Marouli E, et al. Association analyses based on false discovery rate implicate new loci for coronary artery disease. Nat Genet. 2017;49(9):1385-91.

29. Van Der Harst P, Verweij N. Identification of 64 novel genetic loci provides an expanded view on the genetic architecture of coronary artery disease. Circ Res. 2018;122(3):433-43.

30. Clarke GM, Anderson CA, Pettersson FH, Cardon LR, Morris AP, Zondervan KT. Basic statistical analysis in genetic case-control studies. Nat Protoc. 2011; 6(2):121-33

31. Inouye M, Abraham G, Nelson CP, Wood AM, Sweeting MJ, Dudbridge F, et al. Genomic Risk Prediction of Coronary Artery Disease in 480,000 Adults: Implications for Primary Prevention. J Am Coll Cardiol. 2018;72:1883-93.

32. Vujkovic M, Keaton JM, Lynch JA, Miller DR, Zhou J, Tcheandjieu C, et al. Discovery of 318 new risk loci for type 2 diabetes and related vascular outcomes among 1.4 million participants in a multi-ancestry meta-analysis. Nat Genet. 2020;10:26

33. Mahajan A, Taliun D, Thurner M, Robertson NR, Torres JM, Rayner NW, et al. Fine-mapping type 2 diabetes loci to single-variant resolution using highdensity imputation and islet-specific epigenome maps. Nat Genet. 2018; 50(11):1505-13.

34. Mavaddat N, Michailidou K, Dennis J, Lush M, Fachal L, Lee A, et al. Polygenic risk scores for prediction of breast cancer and breast cancer subtypes. Am J Hum Genet. 2019;104(1):21-34.

35. Nielsen JB, Thorolfsdottir RB, Fritsche LG, Zhou W, Skov MW, Graham SE, et al. Biobank-driven genomic discovery yields new insight into atrial fibrillation biology. Nature Genetics. 2018:50:1234-9.

36. Jansen IE, Savage JE, Watanabe K, Bryois J, Williams DM, Steinberg S, et al. Genome-wide meta-analysis identifies new loci and functional pathways influencing Alzheimer's disease risk. Nat Genet. 2019;51(3):404-13.

37. Craig JE, Han X, Qassim A, Hassall M, Cooke Bailey JN, Kinzy TG, et al. Multitrait analysis of glaucoma identifies new risk loci and enables polygenic prediction of disease susceptibility and progression. Nat Genet. 2020:52:160-6.

38. Ramnarine S, Zhang J, Chen LS, Culverhouse R, Duan W, Hancock DB, et al. When does choice of accuracy measure alter imputation accuracy assessments? PLoS One. 2015;10(10):137601

39. Esserman LJ. The WISDOM Study: breaking the deadlock in the breast cancer screening debate. npj Breast Cancer. 2017;3:1-7.

40. MyGeneRank. https://mygenerank.scripps.edu/. Accessed 10 Sept 2020.

41. KardioKompassi. https://kardiokompassi.fi/. Accessed 10 Sept 2020.

42. Kim JO, Schaid DJ, Cooke A, Kim C, Goldenberg BA, Highsmith WE, et al. Impact of a breast cancer (BC) polygenic risk score (PRS) on the decision to take preventive endocrine therapy (ET): The Genetic Risk Estimate (GENRE) trial. J Clin Oncol. 2019;37(15_suppl):1501.

43. Preventive Genomics Clinic. https://www.massgeneral.org/medicine/ treatments-and-services/preventive-genomics-clinic. Accessed $10 \mathrm{Sept}$ 2020.

44. Zhang Y, Qi G, Park JH, Chatterjee N. Estimation of complex effect-size distributions using summary-level statistics from genome-wide association studies across 32 complex traits. Nat Genet. 2018;50:1318-26.

45. Martin AR, Kanai M, Kamatani Y, Okada Y, Neale BM, Daly MJ. Clinical use of current polygenic risk scores may exacerbate health disparities. Nat Genet. 2019;51:584-91. 
46. Atherosclerosis Risk in Communities Study. dbGAP. 2010. https://www.ncbi. nlm.nih.gov/projects/gap/cgi-bin/study.cgi?study_id=phs000280.v6.p1. Accessed 14 Nov 2020.

47. Dias R, Chen S-F. Imputation Accuracy Calculator. Github. https://github. com/TorkamaniLab/imputation_accuracy_calculator. Accessed 14 Nov 2020.

\section{Publisher's Note}

Springer Nature remains neutral with regard to jurisdictional claims in published maps and institutional affiliations.

Ready to submit your research? Choose BMC and benefit from:

- fast, convenient online submission

- thorough peer review by experienced researchers in your field

- rapid publication on acceptance

- support for research data, including large and complex data types

- gold Open Access which fosters wider collaboration and increased citations

- maximum visibility for your research: over $100 \mathrm{M}$ website views per year

At $\mathrm{BMC}$, research is always in progress.

Learn more biomedcentral.com/submissions 